\title{
Heavy Hybrids with Constituent Gluons
}

\author{
Eric S. Swanson ${ }^{1,2}$ and Adam P. Szczepaniak ${ }^{3}$ \\ ${ }^{1}$ Department of Physics, North Carolina State University, Raleigh, North Carolina 27695-8202 \\ 2 Jefferson Laboratory, 12000 Jefferson Avenue, Newport News, VA 23606 \\ 3 Physics Department, Indiana University, Bloomington, Indiana 47405-4202
}

\begin{abstract}
Hybrid meson energies are calculated in the static quark limit with the Dynamical Quark Model (DQM). In the DQM, transverse gluons are represented as effective constituents with a dynamically generated mass. Hybrid masses are determined within the Tamm-Dancoff approximation for the resulting relativistic Salpeter equation. Although the general features of the adiabatic potential surfaces correspond with lattice data, the results disagree on level orderings. Similar problems appear to exist in all constituent glue models of hybrids. We conclude that constituent gluons do not accurately represent soft gluonic degrees of freedom. The steps necessary to correct this deficiency are discussed.
\end{abstract}

Typeset using REVTEX 


\section{INTRODUCTION}

A decade of experimental signals [1] for QCD hybrids (in particular with $J^{P C}=1^{-+}$) has culminated in the claimed observation of three such states at Brookhaven [2] in the last year. The question of the nature of QCD hybrids has thus become topical. Furthermore, lattice gauge calculations are now at the point of accurately determining light hybrid masses. In view of these developments, it is of interest to compare models of strong (low energy) QCD with lattice data to determine their viability and to explicate and guide current experimental efforts.

It is often stated that a hybrid is a hadron consisting of valence quarks and glue. However, one must specify what is meant by the notion of valence glue for this statement to be useful. There are two broad ideas in this regard: it is some sort of string or flux tube [3, [1] or it is an effective constituent confined by a bag [5,6] or potential [7] 9 .

As an example of the importance of choosing correct degrees of freedom, we mention the simple problem of determining the number of components of a constituent gluon. It has been suggested that a massive constituent gluon should be transverse so as to maintain consistency with Yang's theorem [8]. However it was noted that this is inconsistent with the requirements of Lorentz invariance. Thus, for example, $J=1$ glueballs are expected to exist and lattice calculations indicate that they are quite heavy (roughly $3 \mathrm{GeV}$ ) [10]. Such a state may not be constructed from two transverse constituent gluons (Yang's theorem) and therefore may be expected to have a mass of roughly $3 m_{g} \sim 3 \mathrm{GeV}$. However massive vector gluons have no such constraint and one therefore expects them to have a mass of approximately $2 m_{g} \sim 2 \mathrm{GeV}$.

The nature of the appropriate effective degrees of freedom for glue can only be determined by a long process of calculation and comparison with experimental and lattice data. There are, however, a few indications that low energy glue is string-like. Perhaps the most compelling of these are lattice calculations of energy, action, or field densities between static color sources which are reminiscent of flux tubes [11]. An intriguing clue is also provided 
by the spin splittings of heavy quarkonia. It is known that an effective interaction free of long range exchange spin-orbit coupling is needed to reproduce the mass splitting of the ${ }^{3} P_{J}$ heavy quarkonium multiplets. However, an analysis of QCD in the heavy quark limit convincingly demonstrates that obtaining such an effective potential requires that low energy glue must be string-like [12].

Alternatively, pointlike models of low energy glue have a long history, originating with MIT bag model calculations of Barnes [5] and others [6]. Horn and Mandula [0] were the first to consider a potential constituent glue model of hybrids. Their hybrids consisted of constituent quarks and pointlike, massless, spinless, and colorless glue in a nonrelativistic potential model. The confining potential was taken to be linear with a string tension given by the ratio of color Casimir operators: $b_{q g}=9 / 8 b_{q \bar{q}}$. The authors noted that the two body $q \bar{q}$ potential is anti-confining in the color octet channel and has a repulsive Coulomb spike at short distances. They argued that this is unphysical and hence choose to neglect this term in the interaction. It is clear that a great many simplifying assumptions have gone into the construction of this model. It is our purpose to compare a more sophisticated version of the model to lattice data to learn something about these assumptions.

In the following we employ a model field theoretic Hamiltonian of QCD. The model incorporates linear confinement at low energy and evolves into perturbative QCD at high energy. A nontrivial vacuum is used to generate constituent quark and gluon masses. The eigenvalue equation is derived for a $q \bar{q} g$ system where the quarks are static. The resulting adiabatic potential surfaces are then compared to recent lattice results. We conclude that the simple picture of glue as a pointlike constituent particle reproduces the general behaviour of the lattice results but fails to yield the correct level orderings. Furthermore, other models which regard the gluonic degrees of freedom as pointlike (eg., [18], [7]) do not contain sufficient degrees of freedom to generate all of the adiabatic surfaces. Thus constituent glue models appear to fail to describe hybrids. This stands in contrast to string or bag-like models which, although disagreeing on details, capture the rough structure of the lattice data. 


\section{A CONSTITUENT GLUE MODEL OF STATIC HYBRIDS}

\section{A. The Dynamical Quark Model}

The starting point for our description of hybrids is the following model Hamiltonian:

$$
\begin{aligned}
H & =\int d \mathbf{x} \psi^{\dagger}(\mathbf{x})[-i \vec{\alpha} \cdot \vec{\nabla}+\beta m] \psi(\mathbf{x})+\frac{1}{2} \int d \mathbf{x}\left[\left|\mathbf{E}^{A}(\mathbf{x})\right|^{2}+\left|\mathbf{B}^{A}(x)\right|^{2}\right] \\
& +\frac{1}{2} \int d \mathbf{x} d \mathbf{y} \rho^{A}(\mathbf{x}) V(|\mathbf{x}-\mathbf{y}|) \rho^{A}(\mathbf{y})
\end{aligned}
$$

where the color charge density is $\rho^{A}(\mathbf{x})=\psi^{\dagger}(\mathbf{x}) \mathrm{T}^{A} \psi(\mathbf{x})-f^{A B C} \mathbf{A}^{B}(\mathbf{x}) \cdot \mathbf{E}^{C}(\mathbf{x})$ and the potential is given by

$$
V(r)=\frac{\alpha_{s}}{r}-\frac{2 N_{c} b}{N_{c}^{2}-1} r\left(1-\mathrm{e}^{-\Lambda_{U V} r}\right)
$$

and $N_{c}=3$. The quark mass appearing in this Hamiltonian is the current mass. To be phenomenologically successful, constituent quark masses must be generated in some way. This may be achieved by employing a BCS vacuum Ansatz; the gap equation which follows from minimizing the vacuum energy density, $\langle\Omega|H| \Omega\rangle$, (where $|\Omega\rangle$ represents the BCS trial vacuum) gives rise to a low energy constituent quark mass of roughly $200 \mathrm{MeV}$ [13]. A similar calculation in the glue sector yields a gluon dispersion relation which is well-approximated by

$$
\omega(k)=\sqrt{k^{2}+m_{g}^{2} \mathrm{e}^{-k / \kappa}}
$$

with $m_{g}=800 \mathrm{MeV}$ and $\kappa=6.5 \mathrm{GeV}$. One sees that a constituent gluon mass of approximately $800 \mathrm{MeV}$ has been generated [14]. Hadrons are then constructed on top of the BCS vacuum $|\Omega\rangle$ by employing a basis truncation (typically Tamm Dancoff or Random Phase) and solving the resulting Bethe-Salpeter equation. This approach has been used to derive the low lying spectrum of glueballs and agrees remarkably well with lattice data [14]. It

should be noted that the dynamical gluons are transverse so that Yang's theorem holds and the difficulty mentioned in the Introduction does not arise. 
In the following, the parameters of the model are fixed to the $q \bar{q}$ potential derived in the lattice calculation of Juge, Kuti, and Morningstar [15] (see Fig. 3 below). The fit yields $\alpha_{s}=0.29$ and $b=0.24 \mathrm{GeV}^{2}$. The final parameter, $\Lambda_{U V}$ serves as an ultraviolet cutoff on the linear confinement potential. Its value was determined in Ref. [14] by fitting the gluon condensate, and will be set at $4 \mathrm{GeV}$ in the following. We shall consider the static quark limit in the remainder of this work so that quark masses (and the quark sector of the BCS vacuum) become irrelevant.

The model presented here may be considered as a simplified version of the Coulomb gauge QCD Hamiltonian where the effects of nonabelian gauge couplings have been modeled by the linear confinement term. Furthermore, second order transverse gluon exchange is suppressed by the heavy quark masses. In principle, this approach allows the elimination of the ultraviolet scale $\Lambda_{U V}$ via renormalization. A method for achieving this which is appropriate for nonperturbative Hamiltonian-based calculations is described in Ref. [13].

\section{B. Static Hybrids}

In the Tamm Dancoff approximation, hybrids are constructed as $q \bar{q} g$ excitations of the BCS vacuum. For the heavy hybrids considered here, the (anti)quarks serve as static color sources (sinks) and the gluons are constituent particles as described above. We choose to work in the "diatomic molecule" basis because this facilitates comparison with the lattice results of Ref. 15. Thus the hybrid state may be written as

$$
\left|\vec{R} n_{g} ; j_{g} \Lambda \xi\right\rangle=\int d \vec{k} \varphi_{n_{g} j_{g}}(k) \mathcal{D}_{\mu \Lambda}^{j_{g}}(\hat{R}) \mathcal{D}_{\mu \lambda^{\prime}}^{j_{g} *}(\hat{k}) \sqrt{\frac{2 j_{g}+1}{4 \pi}} \chi_{\lambda \lambda^{\prime}}^{\xi} \frac{\mathrm{T}_{\mathrm{ab}}^{\mathrm{A}}}{2} b_{\vec{R} / 2, a}^{\dagger} d_{-\vec{R} / 2, b}^{\dagger} a_{k, A, \lambda}^{\dagger}|\Omega\rangle .
$$

Wigner rotation functions are written as $\mathcal{D}$ in this expression, $R$ is the distance between the $q \bar{q}$ pair, $\lambda$ is the gluon helicity, and $\varphi$ is the radial hybrid wavefunction in momentum space. The gluon polarization wavefunction, $\chi_{\lambda \lambda^{\prime}}^{\xi}$ is given by $\chi_{\lambda \lambda^{\prime}}^{1}=\delta_{\lambda \lambda^{\prime}} / \sqrt{2}$ and $\chi_{\lambda \lambda^{\prime}}^{-1}=$ $\lambda \delta_{\lambda \lambda^{\prime}} / \sqrt{2}$. The two cases, $\xi=1,-1$, represent transverse magnetic and transverse electric hybrids respectively. Finally, $\Lambda$ is the projection of the gluonic angular momentum onto 
the $q \bar{q}$ axis, $j_{g}$ is the total angular momentum of the gluon, and $n_{g}$ labels the radial basis state. We note that employing helicity basis gluon creation operators makes this expression significantly more compact than the canonical basis.

The Salpeter equation which follows from this ansatz and the Hamiltonian in Eqn. (1) may be obtained from the following matrix element:

$$
\begin{aligned}
& \left\langle\vec{R}^{\prime} n_{g}^{\prime} ; j_{g}^{\prime} \Lambda^{\prime} \xi^{\prime}|H| \vec{R} n_{g} ; j_{g} \Lambda \xi\right\rangle=\int d \vec{k} \varphi_{n_{g}^{\prime} j_{g}^{\prime}}^{*}(k) \varphi_{n_{g} j_{g}}(k) \frac{1}{2}\left[\omega(k)+\frac{k^{2}}{\omega(k)}\right] \\
& +\frac{3}{8} \iint d \vec{q} d \vec{k} \varphi_{n_{g}^{\prime} j_{g}^{\prime}}^{*}(k) \varphi_{n_{g} j_{g}}(k) V(k-q)\left[\frac{\omega(k)^{2}+\omega(q)^{2}}{\omega(k) \omega(q)}\left(1+(\hat{q} \cdot \hat{k})^{2}\right)\right] \\
& -\frac{3}{4} \iint d \vec{q} d \vec{k} \varphi_{n_{g}^{\prime} j_{g}^{\prime}}^{*}(q) \varphi_{n_{g} j_{g}}(k) V(k-q)\left(\mathrm{e}^{i \frac{R}{2} \cdot(k-q)}+\mathrm{e}^{-i \frac{R}{2} \cdot(k-q)}\right) \frac{\omega(k)+\omega(q)}{\sqrt{\omega(k) \omega(q)}} \times \\
& \times \mathcal{D}_{\Lambda \lambda^{\prime}}^{j_{g} *}(\hat{k}) \mathcal{D}_{\Lambda^{\prime} \sigma^{\prime}}^{j_{g}^{\prime}}(\hat{q}) \sqrt{\frac{2 j_{g}+1}{4 \pi}} \sqrt{\frac{2 j_{g}^{\prime}+1}{4 \pi}} \chi_{\lambda \lambda^{\prime}}^{\xi} \chi_{\sigma \sigma^{\prime}}^{\xi^{\prime}} \mathcal{D}_{\mu \lambda}^{1}(\hat{k}) \mathcal{D}_{\mu \sigma}^{1 *}(\hat{q}) \\
& +\left(\frac{1}{6} V(R)+\frac{4}{3} V(0)\right) \mathcal{N}_{n_{g}^{\prime} j_{g}^{\prime} n_{g} j_{g}} \delta_{j_{g}^{\prime} j_{g}} \delta_{\Lambda^{\prime} \Lambda} \delta_{\xi^{\prime} \xi}
\end{aligned}
$$

An overall $\delta\left(\vec{R}^{\prime}-\vec{R}\right)$ is understood and $\mathcal{N}_{n_{g}^{\prime} j_{g}^{\prime} n_{g} j_{g}}=\int d k k^{2} \varphi_{n_{g}^{\prime} j_{g}^{\prime}}^{*}(k) \varphi_{n_{g} j_{g}}(k)$ is the wavefunction normalization factor. The two extra Wigner rotation matrices arise from converting the Cartesian basis implicit in Eqn. (1) and in the expression for the color current to the helicity basis, $a_{k i A}=\epsilon_{H}^{i}(k \lambda) a_{k \lambda A}=\mathcal{D}_{m \lambda}^{1}(\phi, \theta,-\phi) \epsilon_{C}^{i}(m) a_{k \lambda A}$, where $\epsilon_{C}$ and $\epsilon_{H}$ are canonical and helicity polarization vectors respectively.

The first term in this expression is the gluon kinetic energy, the second is the gluon self energy, the third is the gluon potential, and the fourth is the $q \bar{q}$ potential and self energies for static quarks in a color octet. The presence of the gluon and quark self energies assures the infrared finiteness of the Salpeter equation. This appears to be a general feature of color singlet states in our approach [14].

The last task is to identify the diatomic quantum numbers used to label the states. In the Jacob-Wick convention the action of parity on glue is given by

$$
P a_{\vec{k}, \lambda, A} P^{\dagger}=\eta_{g}^{P} \mathrm{e}^{-2 i \lambda \phi} a_{-\vec{k},-\lambda, A}
$$

where $\phi$ is the azimuthal angle of $\hat{k}$ and $\eta_{g}^{P}=-1$ is the intrinsic gluon parity. Thus the hybrid states given in Eqn. (4) are eigenstates of gluonic parity with 


$$
P_{g}\left|\vec{R} n_{g} ; j_{g} \Lambda \xi\right\rangle=\xi \eta_{g}^{P}(-)^{j_{g}+1}\left|\vec{R} n_{g} ; j_{g} \Lambda \xi\right\rangle
$$

A reflection of the glue degrees of freedom through a plane containing the $q \bar{q}$ axis leaves the Hamiltonian invariant and when acting on the states it takes $\Lambda \rightarrow-\Lambda$. For $|\Lambda|>0$ one thus has doubly degenerate states. We call this operation $Y$-parity and perform it by setting $\vec{R} \rightarrow R \hat{z}$ and taking $y \rightarrow-y$ which may be achieved by a parity operation followed by a rotation through $\pi$ about the y-axis. The action of $Y$ on a single transverse gluon state, $|k \lambda A\rangle=a_{k \lambda A}^{\dagger}|0\rangle$ is therefore given by,

$$
Y|k \lambda A\rangle=\mathrm{e}^{-i \pi J_{y}} P|k \lambda A\rangle=\eta_{g}^{P} \mathrm{e}^{2 i \lambda \phi}\left|k^{\prime},-\lambda, A\right\rangle
$$

where $\vec{k}^{\prime}=\left(k_{x},-k_{y}, k_{z}\right)$. For the states defined in Eq. (4) one has

$$
Y\left|\vec{R} n_{g} ; j_{g} \Lambda \xi\right\rangle=\xi \eta_{g}^{P}(-)^{\Lambda+1}\left|\vec{R} n_{g} ; j_{g}-\Lambda \xi\right\rangle
$$

where the relations $\mathcal{D}_{\mu-\mu^{\prime}}^{j}\left(k^{\prime}\right)=(-)^{2 j+\mu+\mu^{\prime}} \mathrm{e}^{-2 i \mu^{\prime} \phi} \mathcal{D}_{-\mu \mu^{\prime}}^{j}(k)$ and $\mathcal{D}_{\mu \mu^{\prime}}^{j}(\hat{z})=\delta_{\mu \mu^{\prime}}$ were used. For $\Lambda \neq 0$ the $\mathrm{Y}$-diagonal states may thus be written as

$$
\left|\vec{R} n_{g} ; j_{g}\right| \Lambda\left|\xi ; \eta_{Y}\right\rangle=\frac{1}{\sqrt{2}}\left(\left|\vec{R} n_{g} ; j_{g}\right| \Lambda|\xi\rangle+\eta_{Y}\left|\vec{R} n_{g} ; j_{g}-\right| \Lambda|\xi\rangle\right)
$$

where $\eta_{Y}= \pm 1$ and,

$$
Y\left|\vec{R} n_{g} ; j_{g}\right| \Lambda\left|\xi ; \eta_{Y}\right\rangle=\xi \eta_{g}^{P} \eta_{Y}(-)^{\Lambda+1}\left|\vec{R} n_{g} ; j_{g}\right| \Lambda\left|\xi ; \eta_{Y}\right\rangle
$$

For $\Lambda=0$ we simply have,

$$
Y\left|\vec{R} n_{g} ; j_{g} 0 \xi\right\rangle=-\xi \eta_{g}^{P}\left|\vec{R} n_{g} ; j_{g} 0 \xi\right\rangle
$$

To allow for easy comparison with conventions used elsewhere, we summarize the transformation properties of $\bar{q} q g$ states under total parity, $P=P_{q} P_{g}$, and charge conjugation $C=C_{q} C_{g}$, including the quark degrees of freedom.

$$
\begin{aligned}
& P\left|\vec{R} n_{g} ; j_{g}\right| \Lambda\left|\xi ; \eta_{Y}\right\rangle=\xi \eta_{g}^{P} \eta_{\bar{q} q}^{P}(-)^{j_{g}+1}\left|-\vec{R} n_{g} ; j_{g}\right| \Lambda\left|\xi ; \eta_{Y}\right\rangle \\
& C\left|\vec{R} n_{g} ; j_{g}\right| \Lambda\left|\xi ; \eta_{Y}\right\rangle=\eta_{g}^{C} \eta_{\bar{q} q}^{C}(-)^{S_{\bar{q} q}+1}\left|-\vec{R} n_{g} ; j_{g}\right| \Lambda\left|\xi ; \eta_{Y}\right\rangle
\end{aligned}
$$


where $\eta_{\bar{q} q}^{P}=\eta_{q \bar{q}}^{C}=\eta_{g}^{C}=-1$. The states introduced in Eq. (10) are therefore also eigenstates of combined $P C$, with

$$
P C\left|\vec{R} n_{g} ; j_{g}\right| \Lambda\left|\xi ; \eta_{Y}\right\rangle=\xi \eta_{g}^{P} \eta_{g}^{C}(-)^{j_{g}+S_{\bar{q} q}}\left|\vec{R} n_{g} ; j_{g}\right| \Lambda\left|\xi ; \eta_{Y}\right\rangle
$$

After dividing out the quark portion of $P C$ we are left with

$$
(P C)_{g}\left|\vec{R} n_{g} ; j_{g}\right| \Lambda\left|\xi ; \eta_{Y}\right\rangle=\xi \eta_{g}^{P} \eta_{g}^{C}(-)^{j_{g}+1}\left|\vec{R} n_{g} ; j_{g}\right| \Lambda\left|\xi ; \eta_{Y}\right\rangle
$$

\section{Results}

The Salpeter equation is solved by expanding the radial wavefunction in a complete basis and by diagonalizing the resulting Hamiltonian matrix. The evaluation of the matrix elements is greatly facilitated by performing the angular integrals analytically. Furthermore, we found it expedient to do the remaining numerical integrals with the potential in position space since the integrand is less oscillatory for large argument in this case. The plane waves and potential were expanded in a double series of Wigner functions yielding a total of ten Wigner functions. The angular integral then evaluates to a sum over a product of six Clebsch-Gordon coefficients.

As discussed above eigenstates of the Hamiltonian may be labelled with the projection of the angular momentum onto the $q \bar{q}$ axis, the product of gluonic parity with charge conjugation, $(P C)_{g}$ and the Y-parity eigenvalue. For $\Lambda \neq 0$ the two Y-parity eigenstates are degenerate. States are therefore denoted by $\Lambda_{(P C)_{g}}^{Y}$ where $\Lambda=0,1,2$ are denoted by $\Sigma, \Pi, \Delta$; $(P C)_{g}=\eta_{g}^{P} \eta_{g}^{C} \xi(-)^{j_{g}+1}=g$ or $u$ for even or odd parity respectively; and $Y=\xi \eta_{g}^{P} \eta_{Y}(-)^{\Lambda+1}$ $= \pm$. The total gluonic angular momentum, $j_{g}$ is not a good quantum number. We have found however, that the sum over radial basis states and gluon angular momentum saturates quickly. For example, Fig 1 . shows the (approximately) exact wavefunction for $\Sigma_{g}^{+}$ with $j_{g}=1$ and a variational single Gaussian orbital. The next contribution to the $\Sigma_{g}^{+}$ eigenstate has $j_{g}=3$, this is shown as the dotted line in the figure. Evidently the eigenstate 
is dominated by the lowest gluonic angular momentum component and the single Gaussian approximation is quite accurate. This remains true for all $R$ studied here, with the $j_{g}=3$ component rising to $13 \%$ of the wavefunction for $R=10 \mathrm{GeV}^{-1}$. These observations support traditional approximations made for hybrids: the use of simple Gaussian wavefunctions [19] and the truncation of the adiabatic Schrödinger equation to lowest $j_{g}$ [21].

\section{FIGURES}

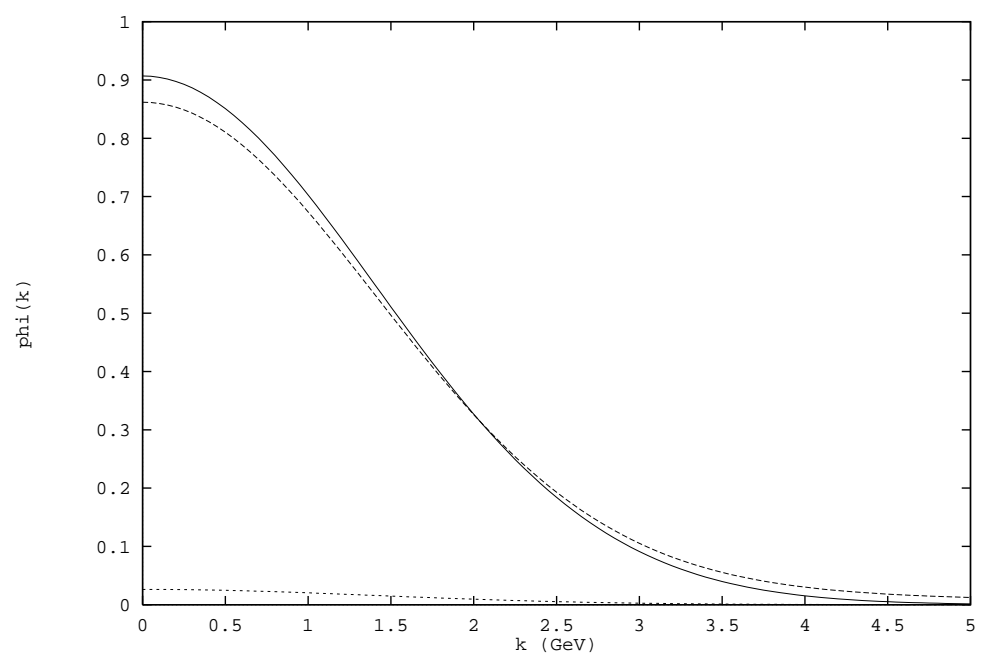

Fig. 1. $\Sigma_{g}^{+}$wavefunctions. Exact result (dashed line), single Gaussian approximation (solid line),

$$
j=3 \text { component (dotted line). }
$$

The results for the gluon spectrum are presented as a function of the static quark separation in Figs. 2 and 3. They are plotted in terms of the lattice scale, $R_{0}=2.32 \mathrm{GeV}^{-1}$ [15] and the potentials have all been normalized by subtracting an overall constant given by $V_{q \bar{q}}\left(2 R_{0}\right)$. In Figs. 2a-c we compare the recent lattice results from Ref. [15] with the predictions of the flux tube model [4. The flux tube model was motivated by the strong coupling limit of the QCD lattice Hamiltonian. It is based upon a nonrelativistic, small oscillation approximation to motion of the colored links in a topological sector where there are no overlapping (color representations of higher dimension) or disconnected links. Under parity (charge) conjugation the spatial (color) orientation of lattice links is reversed, thus the nonrelativistic "beads" of the flux tube model are assumed to flip orientation with respect 
to the position of the quarks under parity and to have positive intrinsic charge conjugation parity.

The lowest solid line in Fig. 2a is a fit to the $q \bar{q}$ potential given by $E(R)=-4 \alpha_{S} /(3 R)+$ $b R+$ const which corresponds to the ground state lattice $\Sigma_{g}^{+}$potential. The other solid lines in Fig. 2 show the flux tube potential as given by

$$
E(R)=b R+\frac{N \pi}{R}\left(1-e^{-f b^{1 / 2} R}\right)
$$

with $f \sim 1$ and $N=\sum_{m=1} m\left(n_{m+}+n_{m-}\right)$. The latter represents the total number of righthanded $\left(n_{m+}\right)$ and left-handed $\left(n_{m-}\right)$ transverse phonon modes weighted by the phonon momentum $(m)$. We note that the authors of Ref. [4 included a Coulomb term in this expression, which is incompatible with the lattice results. The flux tube model predicts the first excited $\Sigma_{g}^{\prime+}$ to be split by $N=2$ from the ground states, and two degenerate $\Sigma_{u}^{+}$and $\Sigma_{u}^{-}$potentials at $N=3$. In the flux tube model the lowest $\Pi$ state is predicted to be the $\Pi_{u}$. It is split from the $q \bar{q}$ ground state by $N=1$ and is followed by the $N=2, \Pi_{g}$ potential. The two lowest $\Delta$ states, $\Delta_{g}$ and $\Delta_{u}$ correspond to $N=2$ and $N=3$ respectively.

The flux tube model fits the first excited state, $\Pi_{u}$ quite well over a wide range of the quark separation. It is, however, the only surface to do so at small distance. Furthermore, this may be a fluke due to the particular choice of the short distance cutoff of the $\pi / R$ term employed in Ref. 四. Alternatively, at large distances, the system is expected to act like some sort of string with an excitation energy given by $\pi / R$. The splittings do indeed appear reasonable for all the surfaces considered. It is, however, disconcerting that the $\Pi$ surfaces diverge from the flux tube model predictions for $r \gtrsim 4 R_{0}$. This must be taken as an indication that the simple flux tube model considerations fail for more complex gluonic configurations.

In Fig.3a-c we plot the results of our calculations for $\Sigma, \Pi$ and the $\Delta$ potentials with the flux tube results (solid lines) for comparison. It is apparent that the $\Pi_{g}$ surface lies below the $\Pi_{u}$ surface while the $\Delta_{u}$ lies below the $\Delta_{g}$. This is opposite to the lattice results, indicating that the model fails to reproduce the expected level orderings. The $\Sigma$ levels are 
also permuted with respect to the lattice calculations at small $R$. We note, however, that the correct level orderings may be reinstated by simply flipping the intrinsic parity of the gluon (set $\eta_{g}^{P}=1$ in the expressions above). The resulting surfaces agree reasonably well with the lattice at small distances. For example, the $\Pi_{u}$ and $\Pi_{g}$ potentials are roughly 1 and 2 at the origin in both calculations. We obtain values of approximately 3 and 3.5 for the $\Delta_{u}$ and $\Delta_{g}$ surfaces at the origin, similar to the lattice values of 2 and 3 respectively. The agreement persists to intermediate $r$, after which it is apparent that the model results approach the linear regime much too quickly with respect to the lattice. This is a strong indication that more degrees of freedom become active as the quark separation increases beyond $r \sim R_{0} \sim 1 / 2 \mathrm{fm}$. This is certainly sensible from the flux tube model point of view, where a large number of degrees of freedom are necessary to construct string phonons.

Our results include the contribution due to direct interaction between the static quarks (the last term of Eqn. (5)). This term was set to zero by Horn and Mandula [7] because it anti-confines and therefore may be expected to not produce a flux tube between the quarks. However, we note that it is responsible for producing a hybrid potential slope equal to that of the $q \bar{q}$ ground state potential, in keeping (roughly) with the lattice data. Thus we have chosen to retain this term. Note however, that this implies that there is a short distance repulsive Coulomb spike which should appear at very short quark separation. The appearance of this spike is, however, unphysical because the hybrid may emit a gluon and convert into a $q \bar{q}$ color singlet and a low lying glueball and this is energetically favorable for small $r$. Equivalently, $V_{\Pi_{u}}>V_{q \bar{q}}+m_{\mathrm{gb}}$ for $r \lesssim 0.2 \mathrm{fm}$. Thus the Coulomb spike mentioned above is irrelevant and one should see, instead, a Coulomb core for small $r$. This effect may be easily incorporated into the model presented here by allowing for coupling to the $q \bar{q} g g$ channel. Notice that no core is visible in the lattice data (especially in $\Pi_{u}$, which is measured down to $r \approx 0.04 \mathrm{fm}$ ). The authors of Ref. [15] are currently examining this issue [20]. 

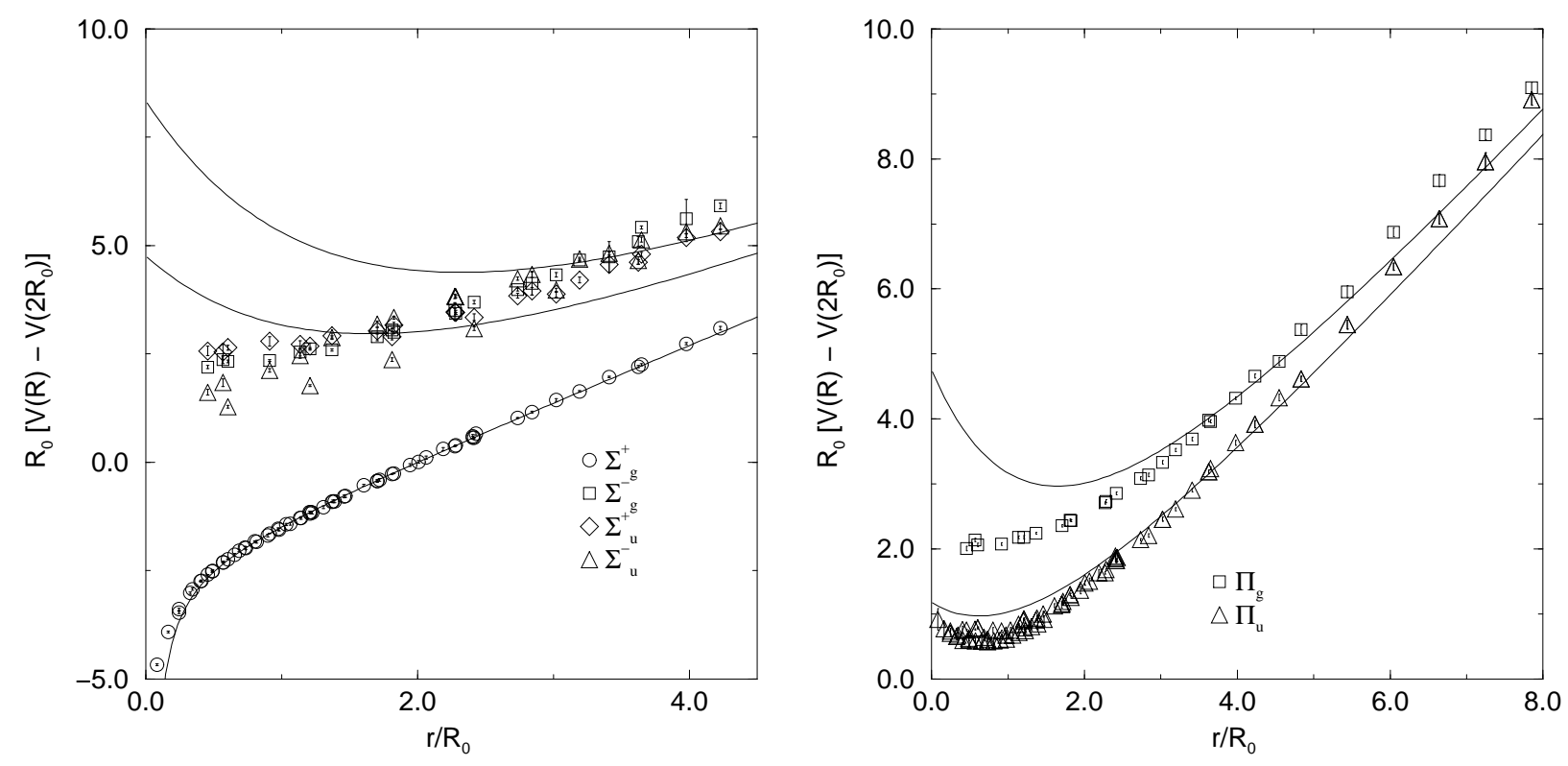

Fig. 2a. $\Sigma$ surfaces. Lattice (symbols) and flux Fig. 2b. П surfaces. Lattice (symbols) and flux tube (lines). tube (lines). 

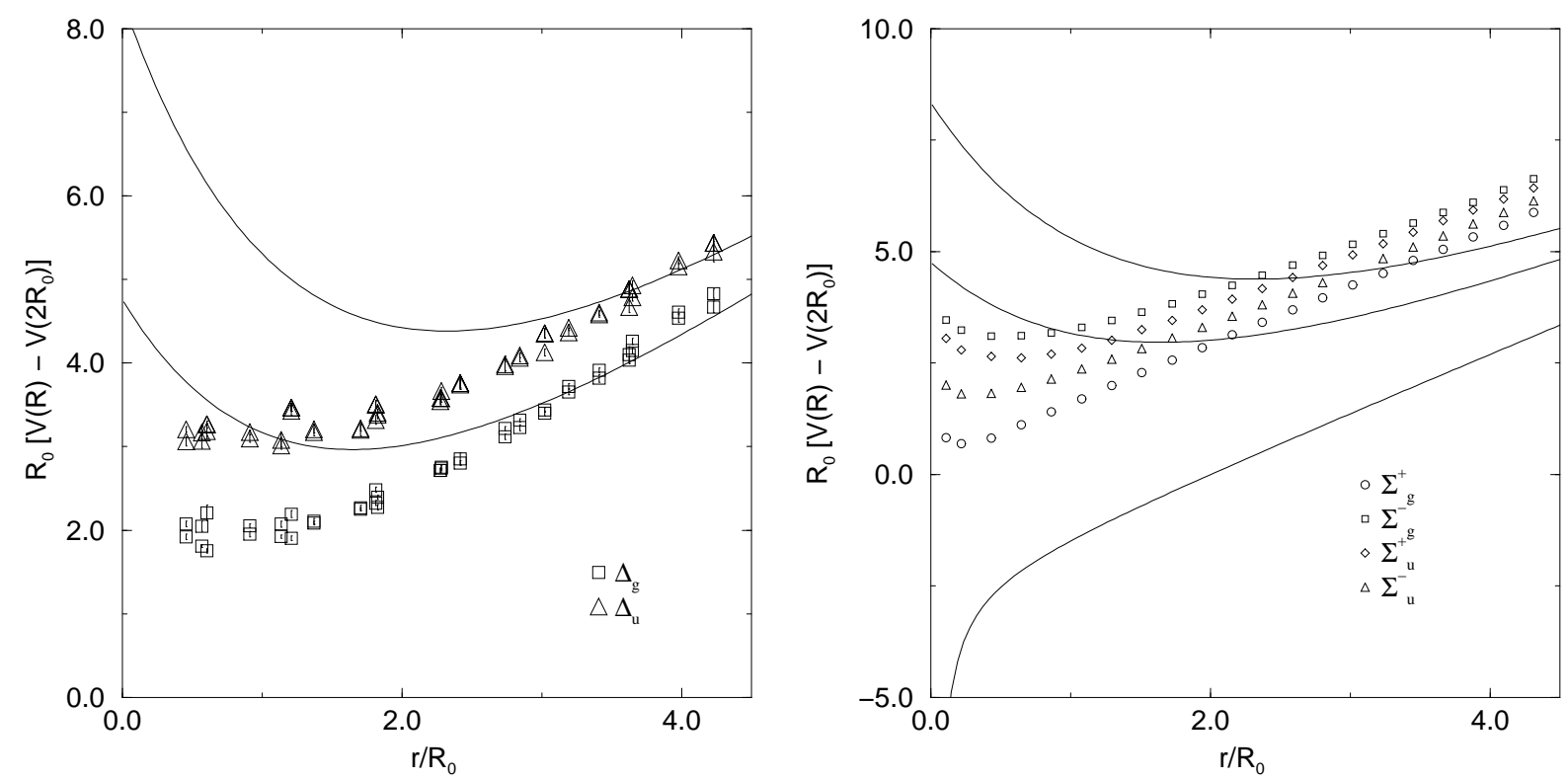

Fig. 2.c. $\Delta$ surfaces. Lattice (symbols) and flux Fig. 3a. $\Sigma$ surfaces. Model (symbols) and flux tube (lines). tube (lines). 

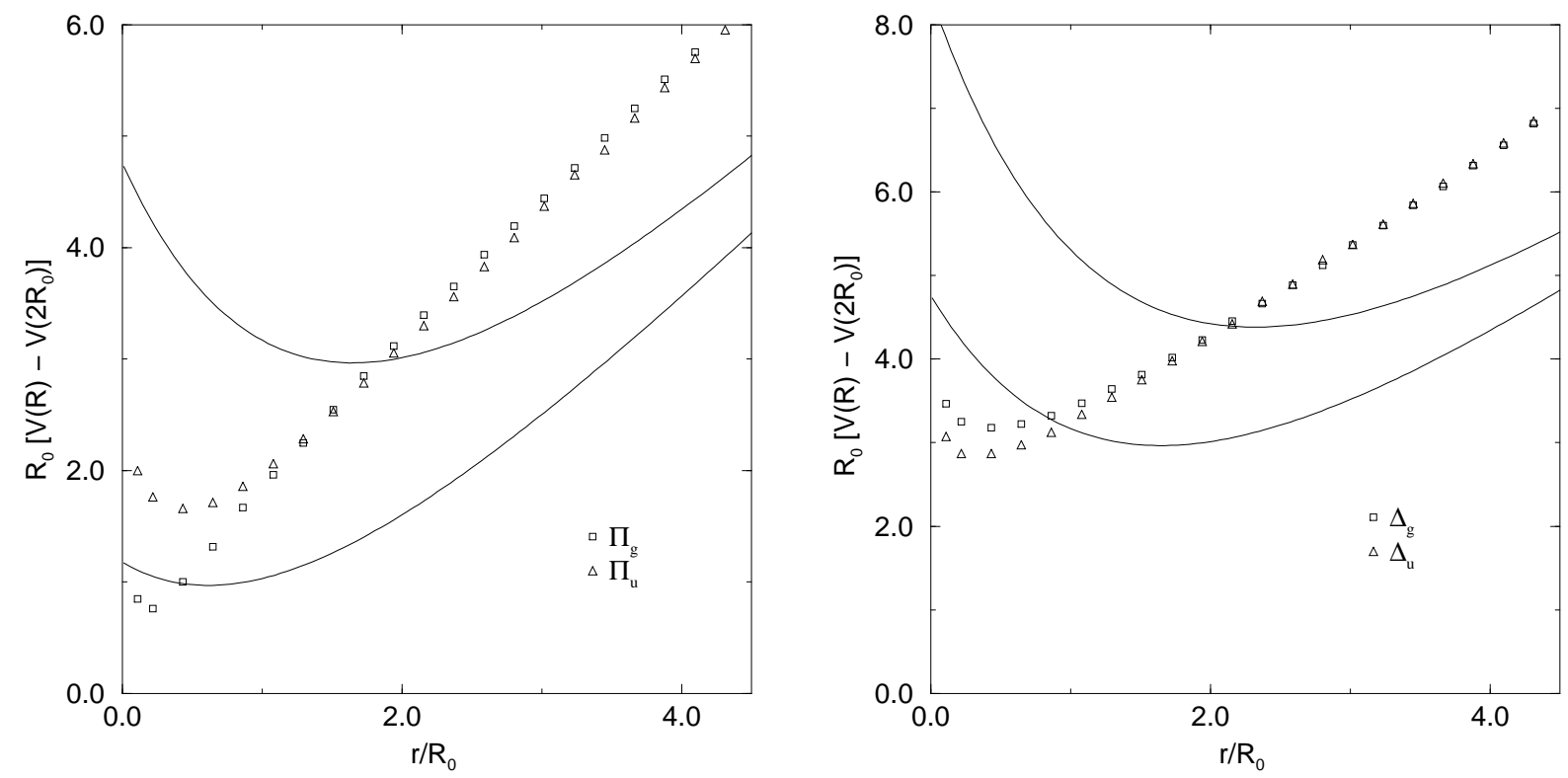

Fig. 3b. $\Pi$ surfaces. Model (symbols) and flux Fig. 3c. $\Delta$ surfaces. Model (symbols) and flux tube (lines). tube (lines).

\section{CONCLUSIONS}

The model presented here agrees moderately well with lattice calculations of adiabatic potential surfaces. However, it disagrees in detail. In particular, it is necessary to ignore the intrinsic parity of the gluon to obtain the expected level orderings. Although one may argue that this is in keeping with the expectations for the parity of a lattice link operator (such as is employed in the development of the Flux Tube model), the lack of consistency is disconcerting. Furthermore, the model predicts surfaces which become linear too quickly with respect to the lattice. We expect this flaw to persist in all constituent glue potential 
models (such as [7] and [18]). This is a strong indication that more degrees of freedom are required to describe soft glue at large interquark separation.

It is important to note that models which employ single spinless gluons do not contain sufficient degrees of freedom to reproduce the potential spectrum. In particular, "single bead" Flux Tube models ( [18]) cannot make $\Pi_{g}$ or $\Sigma_{u}$ states while three dimensional bead models ( [7]) cannot make $\Sigma^{+}$states. Thus including gluon spin is a minimal necessity in this class of models (although the level ordering problem must be overcome as discussed above). We also note that it is possible for spherical bag models to reproduce the lattice calculations at small quark separation, but that they fail at large $R$. Furthermore, Flux Tube model or Nambu-Goto string models [22 reproduce the lattice reasonably well for intermediate to large quark separations, but do not perform well at small $R$ or in detail at large $R$. In particular the potential separation does not appear to be the expected $\pi / R$ and the slopes do not appear to agree. Finally, the bag model of Ref. [6,21] works reasonably well over all quark separations, although problems remain to be resolved in the $\Sigma$ states and for large $R$.

In summary, it appears that some sort of flux tube is required to explain the lattice adiabatic hybrid potential surfaces at large quark separation. This is in keeping with the conclusions of Ref. [12], where flux tubes were required to explain the spin splittings in heavy quarkonia. However, any flux tube model must attempt to incorporate the small $R$ behaviour of the potential surfaces (and the seemingly anomalous behaviour at large $R$ ). This appears to be an indication that different degrees of freedom are required at small distances (as is incorporated in, for example, the bag model of Ref. [6]). Finally, the model presented here (with the gluon parity reversal) works moderately well in describing the potential surfaces and could provide a useful starting point to simple models of gluonic hadron properties (note that the early transition to a linear potential seen in Figs. 3 should not be relevant since the hadron wavefunction is exponentially suppressed in this region). Thus we expect that the success of the previous glueball spectrum calculation [14] in this model (where the gluon parity was irrelevant) was not a fluke. Several benefits of the model are particularly relevant 
to hybrids; light quarks may be easily incorporated, the effects of coupled channels may be examined, and the effects of light quark coupling to virtual transverse or Coulomb gluons may be included. The latter effect is of interest because it is excluded in quenched lattice calculations and may have a substantial effect on the hidden flavor hybrid spectrum.

\section{ACKNOWLEDGMENTS}

The authors are grateful to C. Morningstar for discussions and for providing us the preliminary lattice data of Ref. [15]. This work was supported by the DOE under grants DE-FG02-96ER40944 (ES) and DE-FG02-87ER40365 (AS). 


\section{REFERENCES}

[1] D. Alde et al., Phys. Lett. B205, 397 (1988); H. Aoyagi et al., Phys. Lett. B314, 246 (1993); G.M. Beladidze et al., Phys. Lett. B313, 276 (1993).

[2] D.R. Thompson et al. (E852 Collaboration), Phys. Rev. Lett. 79, 1630 (1997); A. Ostrovidov, Proceedings of Hadron 97, BNL, August, 1997.

[3] H. Nielson and P. Oleson, Nucl. Phys. B61, 45 (1973); Y. Nambu, Phys. Rev. D10, 4262 (1974).

[4] N. Isgur and J. Paton, Phys. Rev. D31, 2910 (1985).

[5] T. Barnes, Caltech Ph.D. thesis, 1977.

[6] P. Hasenfratz, R.R. Horgan, J. Kuti, and J.M. Richard, Phys. Lett. 95B, 299 (1981); T. Barnes, F.E. Close, and F. De Viron, Nucl. Phys. B224, 241 (1983); M. Chanowitz and S. Sharpe, Nucl. Phys. B222, 211 (1983).

[7] D. Horn and J. Mandula, Phys. Rev. D17, 898 (1978).

[8] T. Barnes, Z. Phys. C10, 275 (1981).

[9] J. Cornwall and A. Soni, Phys. Lett. 120B, 431 (1983).

[10] G.S. Bali et al., Phys. Lett. B309, 378 (1993).

[11] G.S. Bali, K. Schilling, and C. Schlichter, Phys. Rev. D51, 5165 (1995).

[12] A.P. Szczepaniak and E.S. Swanson, Phys. Rev. D55, 3987 (1997).

[13] A.P. Szczepaniak and E.S. Swanson, Phys. Rev. D55, 1578 (1997).

[14] A.P. Szczepaniak, E.S. Swanson, C.-R. Ji, and S.R. Cotanch, Phys. Rev. Lett. 76, 2011 (1996).

[15] K. Juge, J. Kuti, and C.J. Morningstar, hep-lat/9709131. 
[16] A.Le Yaouanc, L. Oliver, O. Péne, J-C. Raynal, Z. Phys. C 28, 309 (1985).

[17] S. Ono, Z. Phys. C. 26, 307 (1984).

[18] T. Barnes, F.E. Close, and E.S. Swanson, Phys. Rev. D52, 5242 (1995).

[19] See, for example, P. Page and F.E. Close, Nucl.Phys. B443, 233 (1995); E.S. Swanson and A.P. Szczepaniak, Phys. Rev. D56, 5692 (1997).

[20] C.J. Morningstar, private communication.

[21] K.J. Juge, J. Kuti, and C.J. Morningstar, hep-lat/9709132.

[22] T.J. Allen, M.G. Olsson, and S. Veseli, hep-ph/9804452. 